\title{
mbir: Magnitude-Based Inferences
}

\section{Kyle D Peterson ${ }^{1}$ and Aaron R Caldwell ${ }^{2}$}

1 Sports Science, University of lowa 2 Exercise Science Research Center, University of Arkansas

DOI: $10.21105 /$ joss. 00746

\section{Software}

- Review ¿

- Repository ๘

- Archive ct

Submitted: 06 May 2018

Published: 22 January 2019

\section{License}

Authors of papers retain copyright and release the work under a Creative Commons Attribution 4.0 International License (CC-BY).

\section{Summary}

mbir is an R package (Peterson \& Caldwell, 2018) to provide practitioners and researchers a wholesale approach for deriving magnitude-based inferences from raw data. Magnitudebased inferences, popularized by Hopkins et al (Hopkins WG, Marshall SW, Batterham AM, \& J, 2008), was originally constructed for sport practitioners to derive qualitative interpretations of effect statistics via Microsoft Excel@ spreadsheets (Hopkins, 2018). mbir is now the first open-source solution to such methodology in both R (R Core Team, 2018) and jamovi (jamovi project, 2018).

For a concise description, magnitude-based inferences offer a form of statistical inference in which the parameter is partitioned into three regions corresponding to various qualitative aspects. A quasi-probability (MBI\%) is also assigned to each of these regions, calculated from the assumed distribution of the parameter estimate after being adjusted by the calculated effect size. mbir functions provide qualitative labels to comprise content-rich inferential statements; taking into consideration the level of confidence (possibly, likley, etc), level of magnitude (trivial, small, moderate, etc), as well as the direction of effect (negative, positive).

The primary objective of mbir is to programmatically detect appropriate statistical tests to run in order to drastically curtail the statistical degrees of freedom in an automated fashion. Users simply supply data vectors and allow mbir functions to make statisticallysound decisions, perform respective calculations, and highlight the most suitable interpretations. Figure 1 exemplifies the logic behind a standardized mean difference $(t$-test) function. In this case, establishing whether the distributions deviate from a comparably normal shape and whether homogeneity of variance between vectors is present, will dictate which statistical test to apply to arrive at proper effect statistic. Although such logic are default settings, users also have the freedom to code their own statistical decisions independently, if desired. 


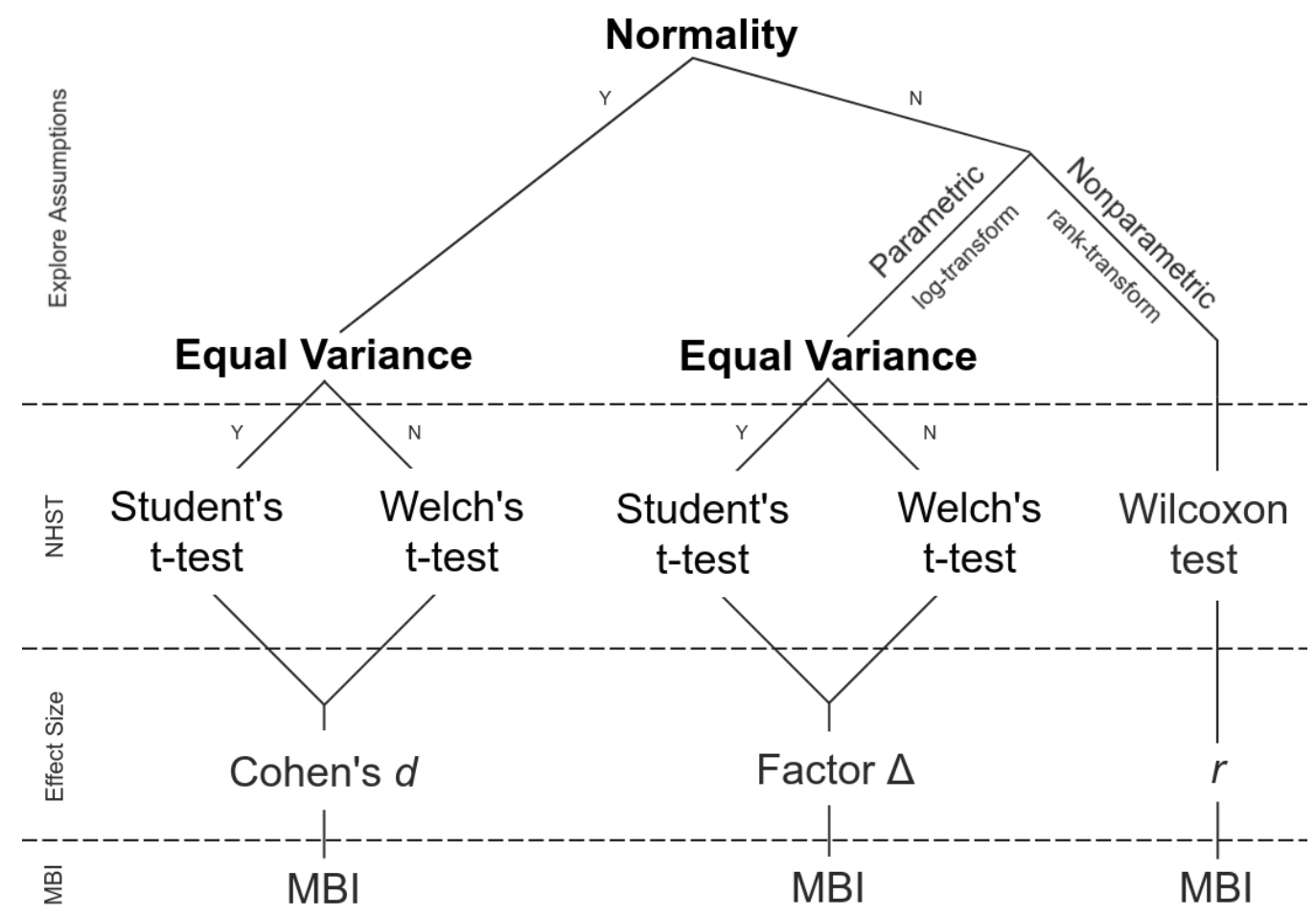

Outside of standard $t$-tests and correlations, mbir offers a collection of strategies for calculating a priori sample size estimations, nonparametric confidence intervals via bootstrap resampling, and a couple ways to analyze individual longitudinal trends via linear regression.

\section{References}

Hopkins, W. (2018). The new view of statistics. Retrieved from http://sportsci.org/ resource/stats/index.html

Hopkins WG, Marshall SW, Batterham AM, \& J, H. (2008). Progressive statistics for studies in sports medicine and exercise science. Medicine and Science in Sport and Exercise, 41(1), 3-13. doi:10.1249/MSS.0b013e31818cb278

jamovi project. (2018). (jamovi version 0.9.). Retrieved from https://www.jamovi.org

Peterson, K., \& Caldwell, A. (2018). mbir: Magnitude-based inferences (R package version 1.3.). Retrieved from http://mbir-project.us

R Core Team. (2018). R: A language and environment for statistical computing. Vienna, Austria: R Foundation for Statistical Computing. Retrieved from https://www. R-project.org/ 